\title{
Detection of Mosaicism in amniotic fluid cultures: A CYT02000 collaborative study
}

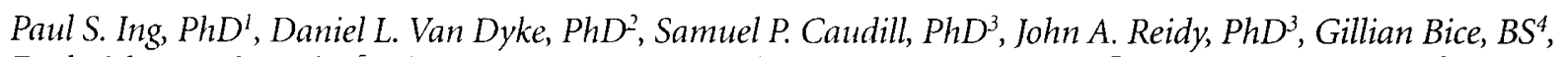
Frederick R. Bieber, PhD ${ }^{5}$, Phillip D. Buchanan, $P h D^{6}$, Andrew J. Carroll, $P h D^{7}$, Sau W. Cheung, $P h D^{8}$, Gordon DeWald, $P h D^{9}$, Roger P. Donahue, $P h D^{10}$, H. Allen Gardner, $M D^{11}$, James Higgins, $P h D^{12}$, Lillian Y.F. Hsu, $M D^{13}$, Mehdi Jamehdor, $P h D^{14}$, Elisabeth A. Keitges, $P h D^{15}$, Caroline H. Laundon, $P h D^{6}$, Frederick W. Luthardt, $P h D^{15}$, James Mascarello, $P h D^{16}$, Kristin M. May PhD $D^{17}$, Jeanne M. Meck, $P h D^{18}$, Cynthia Morton, PhD $D^{5}$, Shiva Patil, $P h D^{19}$,

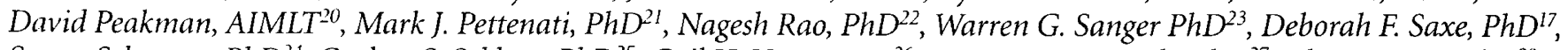
Stuart Schwartz, PhD'24, Gurbax S. Sekhon, PhD ${ }^{25}$, Gail H. Vance, MD ${ }^{26}$, Herman E. Wyandt, PhD'7, Cheng W. Yu, PhD ${ }^{28}$, Julie Zenger-Hain, $P h D^{29}$, and Andrew T. L. Chen, $P h D^{3}$

\begin{abstract}
Purpose: To evaluate the assumptions on which the American College of Medical Genetics (ACMG) Standards and Guidelines for detecting mosaicism in amniotic fluid cultures are based. Methods: Data from 653 cases of amniotic fluid mosaicism were collected from 26 laboratories. A chi-square goodness-of-fit test was used to compare the observed number of mosaic cases with the expected number based on binomial distribution theory. Results: Comparison of observed data from the in situ colony cases with the expected distribution of cases detected based on the binomial distribution did not reveal a significant difference $(P=0.525)$. Conclusions: The empirical data fit the binomial distribution. Therefore, binomial theory can be used as an initial discussion point for determining whether ACMG Standards and Guidelines are adequate for detecting mosaicism. Genetics in Medicine,
\end{abstract} 1999;1(3):94-97

Key words: prenatal diagnosis, cytogenetics, chromosomal mosaicism, guidelines, binomial distribution

\section{INTRODUCTION}

Hook $^{1}$ published tables indicating the number of cells (or colonies in situ) that must be counted to exclude a specified percent mosaicism with $90 \%, 95 \%$, and $99 \%$ confidence. His tables are based on an application of the binomial distribution to the problem of cytogenetically evaluating cultures by counting a prescribed number of metaphases. Use of binomial theory assumes that 1) the probability of mosaicism is constant, 2) the colonies that grow from the cells in the amniotic fluid can be considered independent, ${ }^{2}$ and 3 ) the cells examined represent a random sample of all the cells that could have been collected.

\footnotetext{
From the 'Boys Town National Research Hospital, Omaha, Nebraska; 'Henry Ford Hospital Detroit, Michigan; ${ }^{3}$ Centers for Disease Control and Prevention, Atlanta, Georgia; ${ }^{4}$ Michigan State University, East Lansing; 'Brigham and Women's Hospital/Harvard Medical School, Boston, Massachusetts; ${ }^{6}$ GeneCare Medical Genetics Center, Chapel Hill. North Carolina; 'University of Alabama, Birmingham; "Laboratories for Genetic Services, Inc., Howston, Texas, "Mayo Clinics, Rochester, Minnesota; ${ }^{10}$ University of Miami, Florida; "Oshawa General Hospital, Oshawa, Ontario, Canada; ${ }^{12}$ Butterworth Hospital, Grand Rapids, Michigan; " Prenatal Diagnosis Laboratories of New York City: ${ }^{14}$ Southern California Permanente, Los Angeles, California; ${ }^{15}$ Dynacare, Seattle, Washington; ${ }^{1 n}$ Children's Hospital-San Diego, California; ${ }^{17}$ Emory University, Atlanta, Georgia; ${ }^{18}$ Georgetown University, Washington, DC; "University of Iowa, Iowa City; ${ }^{20}$ Reproductive Genetics Center, PC. Denver, Colorado; "Bowman Gray School of Medicine, ${ }^{22}$ Bowman Gray School of Medicine, Winston-Salem, North Carolina (currently at University California, Los Angeles); ${ }^{23}$ University of Nebraska, Omaha: ${ }^{24}$ Case Western Reserve University, Cleveland, Ohio; ${ }^{25}$ University of Wisconsin-Madison; ${ }^{20}$ Indiana University, Indiana; ${ }^{77}$ Boston University School of Medicine, Massachusetts; ${ }^{28}$ University of Mississippi, Jackson; and ${ }^{29}$ Oakwood Hospital, Dearborn, Michigan

Address correspondence to: Andrew T. L. Chen, Centers for Disease Control, MS-F20, 4770 Buford Highway, NE, Atlanta, GA 30341-3742; E-mail: and@@cdc.gov
}

If a laboratory counts one cell per colony, then 15 cells equals 15 colonies, and in further discussions, we will use the term "cells" to represent colonies in situ.

The 1994 "Standards and Guidelines: Clinical Genetics Laboratories" produced by the Laboratory Practice Committee of the American College of Medical Genetics recommends counting "a minimum of 20 cells, distributed as equally as possible between at least two independently established cultures" (Section E4.1.3.1, p. 13) for the Flask technique and "a minimum of 15 cells from at least 15 colonies ( 10 cells if 15 are not available), distributed as equally as possible between at least two independently established cultures" (Section E4.1.3.2, p. 14) for the in situ technique. A related set of guidelines was published by $\mathrm{Hsu}$ et al. ${ }^{3}$

Laboratory directors today are in a dilemma between the need to increase laboratory efficiency (especially in the current climate of healthcare financing) and the necessity to provide as accurate information as possible to the referring physician and, ultimately, the patient. Two other clinical/laboratory concerns are 1) whether the nonmodal cell line detected is associated with a known (and presumably abnormal) phenotype and 2) whether there is a sufficient percentage of cells with the abnormal karyotype to produce a clinically significant phenotype.

\section{MATERIALS AND METHODS}

We collected data from 26 laboratories to determine whether the observed number of cells required to identify chromosomal mosaicism in amniotic fluid cell cultures agrees with that predicted by binomial theory. Laboratory directors were informed 
of the project first by word of mouth and then via open meetings held during the annual meetings of the American Society of Human Genetics in 1995 and American College of Medical Genetics in 1996. Laboratory directors provided data via two sets of inclusion forms: the CYTO2000 "Matrix" form and the CYTO2000 Amniotic Fluid Mosaicism "Data" Form (available at http://www.faseb.org/genetics/acmg/ cyto2000/prtmf.htm and http://www.faseb.org/genetics/acmg/ cyto2000/prafmf.htm, respectively). The Matrix form asked for the number of true mosaics as defined by the laboratory director and the total number of cultures performed by that laboratory for different tissues between the years 1990 through 1995, inclusive. The Data form asked for information relating to each specific case of amniotic fluid mosaicism in addition to the identification of the lab and case, date and type of study, protocol used, ISCN karyotype, and the total number of cells seen and analyzed. For each cell line reported, we asked for 1) the total number of cells with that karyotype, 2) the cell count number at which the cell line was first detected, and 3) the cell count number at which the cell line was first confirmed. This analysis is concerned with the detection (not the confirmation) of the second cell line.

A note on nomenclature: By definition, Type 3 mosaicism ${ }^{4}$ occurs when two or more cell lines are in separate cultures from a sample and all cell lines must have two or more cells with the same karyotype. Mosaicism is first suspected upon detection of a cell with a different karyotype than the first line (item " 2 ," above). Mosaicism is not confirmed until other cells with the second karyotype are observed (item "3," above).]

A chi-square goodness-of-fit test was used to compare the observed number of mosaic cases detected by the 15 th cell counted with the expected number based on binomial distribution theory. To perform this test for the in situ results, the observed data were first stratified (according to calculated percentage mosaicism) into six groups corresponding to the following percentage point intervals with the indicated medians: (0-10], median $8.00 ;(10-15]$, median $13.33 ;(15-20]$, median 17.86; (20-30), median 25.64; (30-40), median 35.71; and (40-50], median 45. Brackets indicate inclusion of the adjacent number, and parentheses indicate exclusion of the adjacent number. Although different laboratories may have used different stopping rules, the observed number of detected mosaic cases in each stratum (for use in the goodness-of-fit calculations) was chosen as the actual number of mosaic cases detected by the 15th cell counted. (Note: A 15 cell counting rule implies 1 ) if no change in cell line is detected by the 15 th cell counted, then counting stops; or 2) if a different cell line is detected by the 15 th cell counted, counting will continue until a mosaic case is confirmed. Thus, if a confirmed mosaic case was not detected, for example, until the 18th cell counted, this confirmed case was not considered a detected case by the 15 th cell counted. Confirmed cases with less than 15 total cells counted were included, because these cases were detected by the 15 th cell counted.) The theoretic expected number of mosaic cases in each stratum detected by the 15th cell counted (for use in the goodness-offit calculations) was based on binomial theory and the actual number of confirmed cases in the corresponding stratum. Thus, the expected number of mosaic cases in stratum $i(i=1,2, \ldots, 6)$ detected by the 15 th cell counted $\left(\mathrm{N}_{\mathrm{d}(\mathrm{t}(\mathrm{c} \times \mathrm{p},(15))}\right)$ was computed as follows:

$\mathrm{N}_{\mathrm{dit}(\mathrm{cxp})(15 i))}=$

$\left[1-\left(\left(1-P_{\text {Mos(i) }}\right)^{15}+\left(P_{\text {Mus(1) }}\right)^{15}\right)\right] N_{\text {(connirmini) }}$

where $P_{M(\text { os(i) }}$ and $N_{(\text {confimini) }}$ are the observed median proportion mosaicism and the number of confirmed mosaic cases, respectively, for stratum i. Assuming no false positive results, $\mathrm{N}_{\text {(confirm(i)) }}$ will always be less than or equal to the true number ( $\mathrm{N}_{\text {tructii }}$ ) of mosaic cases for stratum $\mathrm{i}$ and the difference between $\mathrm{N}_{\text {(contirm(i) }}$ and $\mathrm{N}_{\text {truc(i) }}$ will increase as $P_{\text {Musii) }}$ decreases. Thus, the chi-square test should be liberal (i.e., more likely to indicate a deviation from the binomial distribution than if $\mathrm{N}_{\text {(ruc }}$ i) were known).

Once we confirmed that the observed data fit a binomial distribution, we performed calculations to estimate the expected true number of mosaic cases in the $\mathrm{i}^{\mathrm{ith}}$ stratum ( $\mathrm{N}_{\text {truetexp(i1) }}$ ) based on the assumption that detected cases were based on 15 cell counts. This estimate was obtained as:

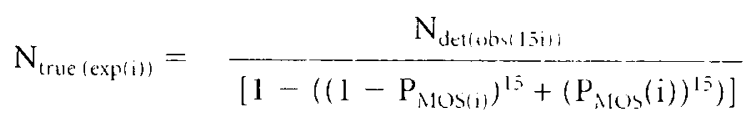

where $N_{\text {detiobs(i) }}$ is the actual number of mosaic cases detected by the 15 th cell counted for stratum $i$, and where $P_{\text {inus }(i)}$ is defined as in equation 1 . Thus, equation 2 assumes that the true mosaic proportion for all cases in a given stratum is equal to the median of the observed mosaic proportions that make up that stratum.

We then used $\mathrm{N}_{\text {truc (ixp(i)) }}$ and $\mathrm{N}_{\mathrm{dctiub}(151) \text { ) }}$ to estimate the percentage of missed mosaic cases in our data set when 15 cells were counted:

$$
\mathrm{PCT}_{\text {(miss(15i)) }}=\frac{100\left[\mathrm{~N}_{\text {truetexp(i)) }}-\mathrm{N}_{\text {detrut)(15i)i }}\right]}{\mathrm{N}_{\text {truetcxp(1)) }}}
$$

We also computed the percentage of missed mosaic cases expected if only 12,10, or 7 cells had been counted as follows:

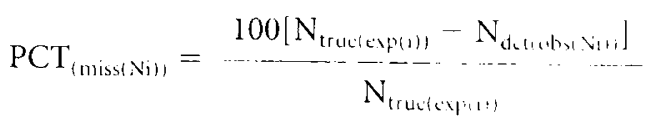

where $\mathrm{PCT}_{(\text {missiNi) }}$ and $\mathrm{N}_{\text {dethobs:in) }}$ represent the percentage of mosaic cases missed and the number of mosaic cases detected by the $\mathrm{N}^{\text {th }}$ cell counted, respectively, for stratum i.

\section{RESULTS}

Twenty-six laboratories provided specific information on 653 cases of amniotic fluid mosaicism via the D)ata form (out of 241,652 total cases). Although the data collection forms requested data from 1990 to 1995, some laboratories included data from other years. In retrospect, we discovered that sume laboratories routinely analyze more than the ACMG standard. In 11 cases, fewer than 15 cells/colonies were analyzed; presumably, all material was utilized; these were included in the data set analyzed because the second line was detected within the first 15 cells/colonies analyzed. In 9 cases, the second cell line was detected after the 15th cell/colony; these were excluded from the analysis.

The average number of cases of amniotic fluid mosaicism per lab was 24.6; the range was between 4 and 59. An average incidence rate of 2.7 cases of mosaicism per 1000 cases cultured 
between 1990 and 1995 (653 cases detected from a total of 241,652 samples cultured) was found; however, there were significant differences between labs (incidence rates ranged from 1.3-6.5 mosaics per 1000 cases, $P<0.0001$ by chi-square goodness-of-fit test). Seventeen cases were excluded from the final analysis due to ambiguous data, duplications of previous studies (confirmation studies), or unconfirmed mosaicism, yielding 636 cases. Almost $94 \%$ of the cases (599) were done between 1990 and 1995; 7 cases (1\%) were done before 1990; and 32 cases (5\%) were done in 1996 and 1997. An average of 32 cells/colonies were analyzed per case; the range was from 7 to 123 . Almost $96 \%$ of cases (611) had only two cell lines. Four hundred forty-two cases $(69.5 \%)$ were analyzed using the in situ method; 92 cases (14.5\%) were analyzed using the flask method; 91 cases $(14.3 \%)$ were analyzed using both methods; and 11 cases $(1.7 \%)$ were analyzed using other methods. A detailed description of the dataset will be the subject of a separate report.

Only the data from cases analyzed using the colony in situ method were sufficient for further analysis. Table 1 displays the number of mosaic cases detected by the 15 th cell counted (column 2) and the number of mosaic cases expected to be detected by the 15 th cell counted (column 3 ) based on binomial theory. When the percentage of mosaicism is low, binomial theory implies that counting 15 cells could result in a substantial proportion of mosaic cases being missed. Thus, our estimates of the expected numbers of detected cases tend to be biased downward for samples with lower percentage mosaicism, because the denominators used to compute the expected numbers are underestimates of the true numbers of mosaic cases. Even with this bias in the table, the observed number of mosaic cases detected by the 15 th cell counted agrees well with that expected by binomial theory ( $P=0.525$ by chi-square goodness-of-fit test).

Table 2 displays the estimated true number of mosaic cases by percentage of mosaicism as well as the estimated percentage of missed mosaic cases expected if only $7,10,12$, or 15 cells had been counted. Based on the results in column 6 , counting only 15 cells should result in very few missed cases if the percentage of mosaicism is greater than $20 \%$. If the percentage of mosaicism is less than $10 \%$, however, as many as $29 \%$ of mosaic cases are likely to be missed if only 15 cells are counted. If only 10 cells are counted,

Table 1

Observed and expected number of mosaic cases detected by fifteenth cell and expected true number of cases by percentage mosaicism

\begin{tabular}{ccc}
\hline $\begin{array}{c}\text { Percentage } \\
\text { mosaicism }\end{array}$ & $\begin{array}{c}\text { Observed" number } \\
\text { of detected cases }\end{array}$ & $\begin{array}{c}\text { Expected" number } \\
\text { of detected cases }\end{array}$ \\
\hline$>0-10$ & 53 & 41 \\
$>10-15$ & 58 & 54 \\
$>15-20$ & 68 & 66 \\
$>20-30$ & 89 & 88 \\
$>30-40$ & 97 & 98 \\
$>40-50$ & 68 & 68 \\
Total & 433 & 415 \\
\hline
\end{tabular}

"Number of mosaic cases detected by the $15^{\text {th }}$ cell counted.

${ }^{b}$ Number of mosaic cases expected to be detected by the $15^{\text {th }}$ cell counted based on binomial theory.
Table 2

Expected percentage of missed mosaic cases ( $f$ or in situ colony method) by percentage mosaicism and number of cells counted

\begin{tabular}{cccccc}
\hline $\begin{array}{l}\text { Percent } \\
\text { mosaicism }\end{array}$ & $\begin{array}{c}\text { Expected }^{a} \text { true } \\
\text { number of cases }\end{array}$ & \multicolumn{4}{c}{$\begin{array}{c}\text { Expected percentage } e^{b} \\
\text { of missed cases }\end{array}$} \\
\hline $\mathrm{N}$ & 75 & 56 & 43 & 35 & 29 \\
$>0-10$ & 67 & 45 & 28 & 19 & 13 \\
$>10-15$ & 73 & 19 & 11 & 8 & 7 \\
$>15-20$ & 91 & 24 & 9 & 7 & 2 \\
$>20-30$ & 98 & 12 & 4 & 2 & 1 \\
$>30-40$ & 69 & 7 & 4 & 4 & 1 \\
$>40-50$ & 473 & 26 & 16 & 12 & 8 \\
$>0-50$ & & 72 & 72 & 12 \\
\hline
\end{tabular}

"The expected true number of mosaic cases is based on the binomial distribution as explained in "Materials and Methods."

"The expected percentage of missed mosaic cases is based on the binomial distribution assuming at least $\mathrm{N}$ cells are counted in each culture.

as many as $43 \%$ of mosaicisms under $10 \%$ are likely to be missed. These results demonstrate that the required number of cells for detection increases as the percentage of mosaicism decreases.

\section{DISCUSSION}

The CYTO2000 process described by Chen ${ }^{5}$ has encouraged multiple laboratories to contribute data to a collaborative effort designed to answer specific questions of common interest. The 26 laboratories participating in this study accounted for about $25 \%$ of the prenatal cases reported in the AGT Directory in $1995 .{ }^{6}$

The data from the 433 cases of mosaicism detected by the 15 th cell counted in cultures using the in situ colony method conform to the theoretical binomial distribution $(P=0.525)$ as described by Hook. ${ }^{1}$ These data confirm that the theoretical expectations are being met in routine practice.

The issue of the number of cells to analyze before mosaicism is detected has been previously addressed. ${ }^{7-9}$ Cheng et al. ${ }^{9}$ observed a $6 \%$ rate of missed mosaics in amniotic fluid mosaicism based on a count of only 10 cells. Discrepancies in their observations and the data presented here might be best explained by the fact that the authors compared the detection rate for a 10-cells-counted cutoff with an estimate of the true number of mosaic cases based on a 15-cell-count standard; our data indicate an average of $8 \%$ of cases of mosaicism are missed when a 15-cell-count standard is used. The observed average of Cheng at al. ${ }^{9}$ of $6 \%$ plus an additional $8 \%$ due to use of a biased standard is in line with the expected average of $16 \%$ when only 10 cells are counted (Table 2, column 4, last row).

Because the observed data in this study does not differ significantly from theoretical values based on the binomial distribution, expected percentages of mosaic cases detected by counting different numbers of cells can be generated for different percentages of mosaicism. The values in Table 3 show these estimated expected percentages. These values have direct relevance to the consequences of changing laboratory standards for the number of cells analyzed in a culture. 
Table 3

Expected percentage of mosaic cases detected for various percentage mosaicisms by number of cells counted

\begin{tabular}{ccccccccc}
\hline $\begin{array}{l}\text { Number of } \\
\text { cells counted }\end{array}$ & \multicolumn{7}{c}{ Percentage mosaicism } & \\
\hline & 5 & 10 & 15 & 20 & 30 & 40 & 50 \\
5 & 23 & 41 & 56 & 67 & 83 & 91 & 94 \\
7 & 30 & 52 & 68 & 79 & 92 & 97 & 98 \\
10 & 40 & 65 & 80 & 89 & 97 & 99 & $>99$ \\
12 & 46 & 72 & 86 & 93 & 99 & $>99$ & $>99$ \\
15 & 54 & 79 & 91 & 96 & $>99$ & $>99$ & $>99$ \\
20 & 64 & 88 & 96 & 99 & $>99$ & $>99$ & $>99$ \\
\hline
\end{tabular}

The focus of this investigation is the likelihood of detecting more than one cell line. However, there are important management issues regarding the clinical consequences of whether the abnormal cell line is the major or minor line. For example, one might expect a different outcome (and advise the patient accordingly) with a result of $80 \% 47,+21$ cells than with a result of $20 \% 47,+21$ cells, although both cases involve $20 \%$ mosaicism. Hook $^{10}$ has discussed this and other issues regarding the diffculties of interpreting mosaicism in prenatal studies.

A separate study of the clinical outcomes of cases of amniotic fluid mosaicism has been initiated by the CYTO2000 group. Data from that study may determine if there are different thresholds of mosaicism percentages that correlate with different clinical outcomes in different mosaic aneuploidies.

We chose to approach the problem in terms of percentage of mosaicism rather than percentage of abnormal cells to make our analysis directly comparable with the theoretical analysis usually used to determine the number of cells that should be counted to exclude mosaicism. 'It can be observed from Hook ${ }^{10}$ that the difference between the two approaches is minimal.

One major factor driving this investigation was the issue of cost savings for laboratories in reduced time associated with analyzing fewer cells. These savings in operating costs (ability to analyze more cases with the same personnel) must be balanced by the additional human and legal costs potentially associated with missing cases of mosaicism by analyzing fewer cells.

\section{CONCLUSIONS}

The CYTO2000 process is one that is capable of collecting collaborative data to answer specific questions of direct interest to cytogeneticists. The observed data from 433 cases of mosaicism detected by the 15 th cell counted in cultures using the in situ colony method fit the theoretical values of the bino- mial distribution. Because these observed values conform to the theoretical values, expected percentages of mosaic cases detected by counting different numbers of cells can be generated for different percentages of mosaicism. These expected percentages can be used as the initial discussion points for determining whether ACMG Standards and Guidelines for the number of cells routinely counted in the laboratory are adequate for detecting mosaicism and the consequences of changing those standards. Two other factors must be taken into consideration. These include the specific chromosomal abnormality as well as the percentage of cells with that karyotype and any clinical information available (e.g., abnormal ultrasound or maternal screening results, positive family history, etc.).

Finally, additional studies have been initiated by the CYTO2000 group to gather data to correlate clinical outcomes by percentages of mosaicism and type of aneuploidy. These studies should provide data on the relative need to vigorously pursue the determination of the actual percentage of mosaicism for different chromosome aneuploidies.

\section{Acknowledgments}

The authors wish to thank Drs. Ernie Hook and Muin Khoury for comments and guidance in the preparation of this paper. We also acknowledge the contributions to the data set by Patrick D. Storto at Michigan State University, Nyla A. Heerema, formerly of Indiana University and currently at the Hughes Institute, Roseville, $\mathrm{MN}$, and all of the laboratory personnel in all of the labs who contributed to the identification of these cases.

\section{References}

1. Hook EB. Exclusion of chromosomal mosaicism: Tables of $90 \%, 95 \%$, and $99 \%$ con fidence limits and comments on use. Am J Hum Genet 1977;29:94-97.

2. Featherstone T, Cheung SW, Spitznagel E, Peaknan D. Exclusion of chromosomal mosaicism in amniotic fluid cultures: Determination of number of colonies needed for accurate analysis. Prenat Diagn 1994;14:1009-1017.

3. Hsu LY, Kaffe S, Jenkins EC, Alonso L, Benn PA, David K, Hirschhorn K, Lieber E, Shanske A, Shapiro LR, et al. Proposed guidelines for diagnosis of chromosome mosaicism in amniocytes based on data derived from chromosome mosaicism and pseudomosaicism studies. Prenat Diagn 1992;12(7):555-73.

4. Hsu LYF, Perlis TE. United States survey on chromosome mosaicism and pseudomosaicism in prenatal diagnosis. Prenat Diagn 1984;4:97-130.

5. Chen ATL, Ing PS, Reidy IA, Schwartz S, Vance GH, Van Dyke DL. The ACMG CYTO2000 subcommitec? Genct Med 1998;1(1):67.

6. Knutson T, editor. AGT International Laboratory Directory; 1996-1997 Edition. 1997

7. Zenger-Hain J. Stupca P, Kramer D, Myrick P. De Wald GW, Van Dyke DL. The number of cells analyzed to detect mosaicism: A two laboratory study. Am J Hum Gonet 1992;52:A80.

8. Zenger-Hain IL, Stupca P, Kramer D, De Wald GW, Van Dyke DL. The number of cells analyzed to detect mosaicism in amniotic fluid cultures: A two laboratory study First Annual Meeting of the American College of Medical Genetics, 1994; p. 114.

9. Cheng EY, Luthy DA, Dunne DFB, Luthardt RW, Disteche CM. Is the 15-in situ protocol necessary to detect amniotic fluid mosaicism? Am / Obstet Gynecol 1995;173:1025-1030

10. Hook EB. Prevalence, risk, and recurrence. In: Brock DH, Rodeck CH. Fergusen-Smith MA, editors. Prenatal Diagnosis and Screening. New York: Churchill Livingston,
1992:351-392. 